\title{
SYK is activated by mutated MYD88 and drives pro-survival signaling in MYD88 driven B-cell lymphomas
}

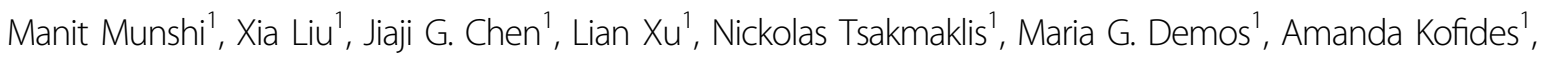 \\ Maria Luisa Guerrera', Cristina Jimenez", Gloria G. Chan', Zachary R. Hunter $\mathbb{D}^{1,2}$, M. Lia Palomba ${ }^{3}$, \\ Kimon V. Argyropoulos ${ }^{3}$, Kirsten Meid ${ }^{1}$, Andrew Keezer ${ }^{1}$, Joshua Gustine ${ }^{1}$, Toni Dubeau ${ }^{1,2}$, Jorge J. Castillo (D) ${ }^{1,2}$, \\ Christopher J. Patterson ${ }^{1}$, Jinhua Wang ${ }^{4}$, Sara J. Buhrlage ${ }^{4}$, Nathanael S. Gray ${ }^{4}$, Steven P. Treon ${ }^{1,2}$ and Guang Yang ${ }^{1,2}$
}

\begin{abstract}
Activating MYD88 mutations promote pro-survival signaling through BTK and HCK, both targets of ibrutinib. Despite high response rates, complete responses to ibrutinib are lacking, and other MYD88 triggered pro-survival pathways may contribute to primary drug resistance. B-cell receptor (BCR) signaling has been observed in lymphomas driven by mutated MYD88, even without activating the BCR pathway mutations. We identified activated SYK (p-SYK), a component of BCR in complex with MYD88 in MYD88-mutated WM and ABC DLBCL lymphoma cells. Confocal microscopy confirmed co-localization of MYD88 with SYK in MYD88-mutated cells. Knockdown of MYD88 or use of a MYD88 signaling inhibitor abrogated SYK activation, while expression of mutated but not wild-type MYD88 amplified p-SYK in MYD88-mutated and wild-type lymphoma cells. Knockdown of SYK or use of inhibitors targeting SYK blocked p-STAT3 and p-AKT signaling in MYD88-mutated cells. Cell viability analysis showed that combining ibrutinib and SYK inhibitors triggered synthetic killing of MYD88-mutated lymphoma cells. Our findings extend the spectrum of mutated MYD88 pro-survival signaling to include SYK directed BCR cross talk in MYD88-mutated lymphomas. Targeting SYK in combination with ibrutinib produces synthetic lethality, providing a framework for the clinical investigation of ibrutinib with SYK inhibitors in MYD88-mutated lymphomas.
\end{abstract}

\section{Introduction}

Activating mutations in MYD88 promote Myddosome self-assembly and trigger Toll-like receptor (TLR) and IL1 receptor (IL-1R) signaling ${ }^{1-5}$. Downstream components of mutated MYD88 signaling include BTK and IRAK4/ IRAK1 that trigger NFKB pro-survival signaling, as well as HCK transactivation that activates BTK itself, AKT and ERK $^{1-6}$. Both BTK and HCK are targets of ibrutinib, a pleiotropic kinase inhibitor that is active in patients with

Correspondence: Steven P. Treon (steven treon@dfci.harvard.edu) 'Bing Center for Waldenstrom's Macroglobulinemia, Dana Farber Cancer Institute and Harvard Medical School, Boston, MA, USA

${ }^{2}$ Department of Medical Oncology, Dana Farber Cancer Institute and Harvard Medical School, Boston, MA, USA

Full list of author information is available at the end of the article
MYD88-mutated B-cell malignancies, including Waldenstrom's macroglobulinemia (WM), ABC subtype of diffuse large B-cell lymphoma (DBLCL), and primary CNS lymphoma $(\mathrm{PCNSL})^{7-10}$. Despite high rates of response to ibrutinib, complete responses are lacking, and other mutated MYD88 triggered pathways may contribute to sustained pro-survival signaling and primary drug resistance.

Activation of B-cell receptor (BCR) signaling is common among MYD88-mutated B-cell malignancies. Activating $C D 79 A / B$ mutations are frequently present in $\mathrm{ABC}$ DLBCL and PCNSL and can trigger SYK-mediated downstream signaling ${ }^{2,11,12}$. In contrast to these aggressive entities, $C D 79 A / B$ activating mutations are uncommon in WM with a reported incidence of $3-12 \%$, and 
have been associated with transformed WM disease ${ }^{13-16}$. Despite the absence of activating BCR mutations, active $\mathrm{B}$-cell receptor $(\mathrm{BCR})$ signaling has been observed in WM and $A B C$ DLBCL patients, and could contribute to prosurvival signaling ${ }^{17-19}$. We therefore have sought to clarify whether mutated MYD88 could account for chronic BCR signaling in MYD88-mutated lymphoma cells. While BTK, a component of the BCR signalosome is triggered by mutated MYD88, our previous work showed that the upstream BCR signaling member SYK was activated in MYD88-mutated WM patient cells ${ }^{17}$. Moreover, the SYK inhibitor fostamatinib triggered apoptosis of MYD88-mutated WM cells, suggesting an important function for SYK in MYD88 pro-survival signaling ${ }^{20}$. We therefore investigated a role for SYK as a mediator of BCR activation in MYD88-mutated lymphomas. Knowledge of MYD88-directed SYK signaling could prompt development of novel therapeutic approaches aimed at extinguishing other routes for MYD88-directed pro-survival signaling beyond ibrutinib and other BTK inhibitors.

\section{Methods}

\section{Cell lines and reagents}

MYD88 L265P $\left(\right.$ MYD88 $\left.{ }^{L 265 P}\right)$ expressing WM (BCWM.1 and MWCL-1), ABC DLBCL (TMD8, HBL-1, OCI-Ly3) and wild-type MYD88 (MYD88 $\left.{ }^{W T}\right)$ GCB DLBCL (OCI-Ly7, OCI-Ly19), Burkitt's lymphoma (Ramos), as well as multiple myeloma (RPMI-8226) cells were used in the studies. The identities of the cell lines used in this study were confirmed via STR profiling with GenePrint $^{\circledR} 10$ System (Promega, Madison, WI) at The Molecular Diagnostic Laboratory at Dana-Farber Cancer Institute. All cell lines are routinely tested to exclude mycoplasma contamination. TMD8 (Y196H) and HBL-1 (Y196F) carry CD79B activating mutations, while OCILY3 is homozygous for $M Y D 88^{L 265 P}$. All cell lines were cultured as previously described ${ }^{5,6}$. The BTK inhibitor ibrutinib, and SYK inhibitors tamatinib (R406), the active metabolite of fostamatinib ${ }^{21}$, and entospletinib (GS9973) 22 were obtained from Selleck Chemicals (Houston, TX). MYD88 inhibitor and control peptides were obtained from Novus Biologicals (Littleton, CO), and used as before in signaling experiments ${ }^{1,5}$.

\section{Patient samples and treatments}

Mononuclear cells from freshly obtained WM patient's bone marrow (BM) aspirates were isolated using FicollPaque $^{\mathrm{TM}}$ PLUS Media (GE Healthcare ${ }^{\mathrm{TM}}$ ) and treated with either ibrutinib, SYK inhibitor tamatinib or entospletinib, or combination of ibrutinib and a SYK inhibitor. Apoptosis analyses were performed on MYD88 genotyped CD19-gated lymphoplasmacytic cells (LPCs) following overnight treatment of $\mathrm{BM}$ mononuclear cells, as previously described ${ }^{5,6,23}$. Subject participation was approved by the Harvard Cancer Center/Dana-Farber Cancer Institute Institutional Review Board, and all participants provided written consent for sample use.

\section{Lentiviral knockdown and expression studies}

Knockdown of endogenous MYD88 in MYD88 ${ }^{L 265 P}$ expressing BCWM.1 WM and MYD $88^{L 265 P}$ and $C D 79 B^{Y 196 H}$ expressing TMD8 cells was undertaken, as well as expression of MYD88 ${ }^{\mathrm{WT}}$ or MYD88 ${ }^{\mathrm{L} 265 \mathrm{P}}$ proteins in BCWM.1, OCI-Ly7 or Ramos cells following lentiviral transduction as previously described ${ }^{5,6}$. Knockdown of SYK was performed using lentiviral vector system as previously described that targeted the following sequences: shRNA-1 (5'-GGGAAGAATCTGAGCAAAT-3'); shRNA-2 (5'-GGATCAAAGACAAATGGAA-3'). Following lentiviral transduction on day 5 , SYK knockdown cells were selected by flow sorting of GFP-positive cells and analyzed in cell viability and signaling studies.

\section{Signaling studies}

PhosFlow and immunoblotting studies were performed as previously described ${ }^{5,6}$ using antibodies to p-SYK (Y525/Y526) (R\&D Systems, MN); p-BTK(Y223), pIRAK1(T209) (Abcam, MA); SYK, p-STAT3(Y705), STAT3, p-AKT(S473), AKT, BTK, IRAK1, p-IRAK4 (T345/S346), IRAK4, and Alexa Fluor 647-conjugated pSYK(Y525/Y526) (Cell Signaling Technologies, MA). Alexa Fluor ${ }^{\circledast}$ 488-conjugated $\alpha$-tubulin antibody (Cell Signaling Technologies) was used as an internal control for p-SYK levels among different cell lines. GAPDH antibody (Santa Cruz Biotechnology, TX) was used as a loading control for immunoblotting. Cell lines or WM patient BM mononuclear cells were treated with inhibitors for $1-2 \mathrm{~h}$ before signaling studies.

\section{Co-immunoprecipitation experiments}

Co-immunoprecipitation studies were performed as previously described ${ }^{5}$ using anti-MYD88 antibody (Santa Cruz Biotechnology) and SYK, p-SYK(Y525/Y526) antibodies (Cell Signaling Technologies). Briefly, cells were lysed with Co-IP buffer (Thermo Fisher Scientific) supplemented with $1 \mathrm{mM}$ sodium orthovanadate, $10 \mathrm{mM} \mathrm{NaF}$, $1 \times$ protease inhibitors cocktail for $15 \mathrm{~min}$ on ice, and then centrifuged at $2600 \times g$ for $5 \mathrm{~min}$. Supernatants $(2 \mathrm{mg}$ total protein) were incubated with $2-4 \mu \mathrm{g}$ of antibodies at $4{ }^{\circ} \mathrm{C}$ for $30 \mathrm{~min}$, followed by incubation with protein A/Gcoated magnetic beads (EMD Millipore) for another $30 \mathrm{~min}$ at $4{ }^{\circ} \mathrm{C}$. After samples were washed four times with ice-cold lysis buffer on a magnetic stand, proteins were eluted using SDS-PAGE loading buffer for further analysis.

\section{Immunofluorescence staining and confocal microscopy}

BCWM.1, MWCL-1, and TMD8 cells were spun onto glass slides with Cytospin ${ }^{\mathrm{TM}} 4$ Cytocentrifuge (Thermo 
Fisher Scientific) at $800 \mathrm{rpm}$ for $5 \mathrm{~min}$. Cells were fixed with 4\% paraformaldehyde in PBS for 20 min, washed twice with PBS, permeabilized with $0.25 \%$ Triton X-100 in PBS for $15 \mathrm{~min}$, and blocked with blocking buffer (5\% BSA and 0.1\% Triton X-100 in PBS) for $1 \mathrm{~h}$. Cells were then incubated with anti-MYD88-Alexa Fluor 488 and anti-SYK-Alexa Fluor 647 antibodies (Santa Cruz Biotechnology) overnight at $4{ }^{\circ} \mathrm{C}$. The slides were washed five times with $1 \times$ PBS and counterstained with mounting media containing DAPI (4',6-diamidino-2-phenylindole dihydrochloride) (Thermo Fisher Scientific) and imaged using Leica SPE Confocal Microscope (Leica Microsystems) using an ACS APO 63×/ 1.30 oil immersion lens. Images were analyzed using Leica Application Suite X software.

\section{Cytotoxicity studies}

The CellTiter-Glo ${ }^{\circledast}$ Luminescent cell viability assay (Promega, Madison, WI) was used to assess the dose response of inhibitors alone or in combination ${ }^{5,6}$. Cells were seeded into 384 or 96-well plates with the EL406 Combination Washer Dispenser (BioTek Instruments, Inc.), and inhibitors were injected into culture media with the JANUS Automated Workstation (PerkinElmer Inc., Waltham, MA, USA). Cells were incubated with inhibitors for $72 \mathrm{~h}$ at $37^{\circ} \mathrm{C}$. Luminescent measurements to assess cell viability were performed using the 2104 Envision $^{\circledR}$ Multilabel Reader (PerkinElmer Inc.). Drug interactions were assessed by CalcuSyn 2.0 software (Biosoft, Cambridge, UK) based on Chou $\mathrm{TC}^{24}$.

\section{Reproducibility and statistical analysis}

Sample size of experiments and the number of experiments performed are depicted in the figure legends. The statistical significance of differences was analyzed using one-way ANOVA with Tukey's multiple comparisons test by Prism software. Differences were considered significant when $p<0.05$. Error bars denote standard deviation.

\section{Results}

SYK is activated in MYD88-mutated lymphoma cells, and its activation is regulated by MYD88

A cross cell line comparison of the phosphorylation levels of SYK (pY525-pY526) showed higher levels of activated SYK in MYD88-mutated versus wild-type cell lines, while ABC DLBCL cells (TMD8, HBL-1) with both $M Y D 88$ and $C D 79 B$ mutations expressed higher levels of phosphorylated SYK versus MYD88 only mutated WM cells (Fig. 1a). The highest p-SYK levels were observed in OCI-Ly3 ABC DLBCL cells that are homozygous for $M Y D 88^{L 265 P}$ (Fig. 1a). More pronounced SYK phosphorylation was also identified in primary MYD88mutated WM cells when compared with healthy donor peripheral B cells (Fig. 1b). We next performed PhosFlow studies for SYK in MYD88-mutated lymphoma cells following treatment with a MYD88 inhibitor or control peptide. Akin to our previous findings for NFkB signaling ${ }^{1,5}$, these studies also showed that phosphorylation of SYK at Tyrosine 525 and 526 (pY525-pY526) was reduced following treatment with a MYD88 peptide inhibitor in both MYD88-mutated WM and $\mathrm{ABC}$ DLBCL cells (Fig. 1c). Noteworthy, SYK phosphorylation was more robustly reduced in the WM cell lines (BCWM.1, MWCL-1), as well as the ABC DLBCL cell line OCI-Ly3 that carry only the MYD $88^{L 265 P}$ mutation, but negligible in ABC DLBCL cells (TMD8, HBL-1) with both $M Y D 88$ and $C D 79 B$ activating mutations (Fig. 1c). Treatment of primary MYD88-mutated WM cells with the MYD88 peptide inhibitor also blocked SYK phosphorylation (Fig. 1d).

To further explore the possibility that SYK was activated by mutated $M Y D 88$, we performed a knockdown of MYD88 in MYD88-mutated BCWM.1 WM cells and $M Y D 88 / C D 79 B$ mutated TMD8 ABC DLBCL cells. While these experiments showed a marked reduction in phosphorylation of SYK (pY525-pY526), and the SYK downstream components STAT3 (pY705) and AKT (pS473) in BCWM.1, these changes were not observed in TMD8 cells, consistent with our prior findings using MYD88 inhibitor peptides (Fig. 2a). As shown in our prior studies, p-BTK (Y223) expression was decreased by MYD88 knockdown in both BCWM.1 and TMD8 cells ${ }^{5}$. Importantly, the expression of MYD88 ${ }^{\mathrm{L} 265 \mathrm{P}}$ but not wild-type MYD88 increased phosphorylated SYK in MYD88mutated BCWM.1, as well as in MYD88 wild-type OCILy7 and Ramos cell lines (Fig. 2b).

\section{Activated SYK is a component of the "Myddosome" signaling complex in MYD88-mutated lymphoma cells}

Since SYK was activated by mutated MYD88, we next sought to clarify if SYK and p-SYK were present in the "Myddosome" signaling complex in MYD88-mutated lymphoma cells. We therefore performed coimmunoprecipitation (Co-IP) experiments using a MYD88-binding antibody, as well as reverse Co-IP experiments using both SYK and p-SYK antibodies in MYD88-mutated BCWM.1 and MYD88 wild-type Ramos cells. The MYD88 antibody effectively pulled down SYK in Co-IP experiments, and both SYK and pSYK antibodies pulled down MYD88, with more robust pulldown of MYD88 by activated SYK in MYD88mutated BCWM.1 cells (Fig. 3a). Conversely, both SYK and p-SYK antibodies failed to pull down MYD88 in MYD88 wild-type Ramos cells (Fig. 3a). As expected, the IP of p-SYK did not reveal SYK itself in line with the results shown in Fig. 1a that showed lack of p-SYK in MYD88 wild-type cells. Further to these findings, we performed cellular co-localization experiments using immunofluorescent (IF) with antibodies for MYD88 and 


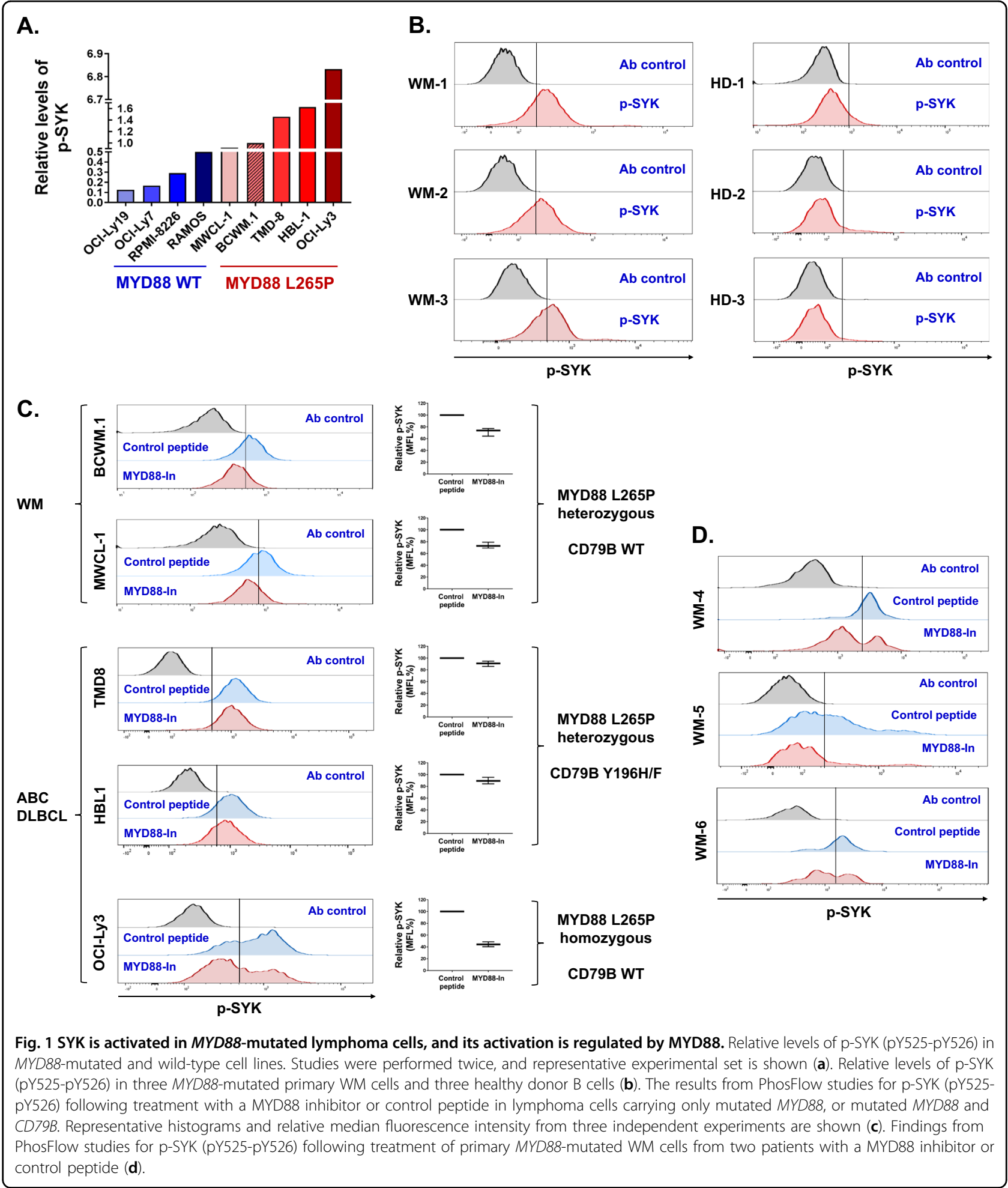

SYK in both MYD88-mutated and wild-type lymphoma cells. By confocal microscopy, IF staining showed that MYD88 and SYK co-localized in the cytoplasm with a punctate staining pattern in MYD88-mutated BCWM.1,
MWCL-1 and TMD8 cells (Fig. 3b), while the MYD88 showed less punctate staining pattern and not co-localized with SYK in MYD88 wild-type Ramos, OCILy7, and OCI-Ly19 cells (Fig. 3b). 


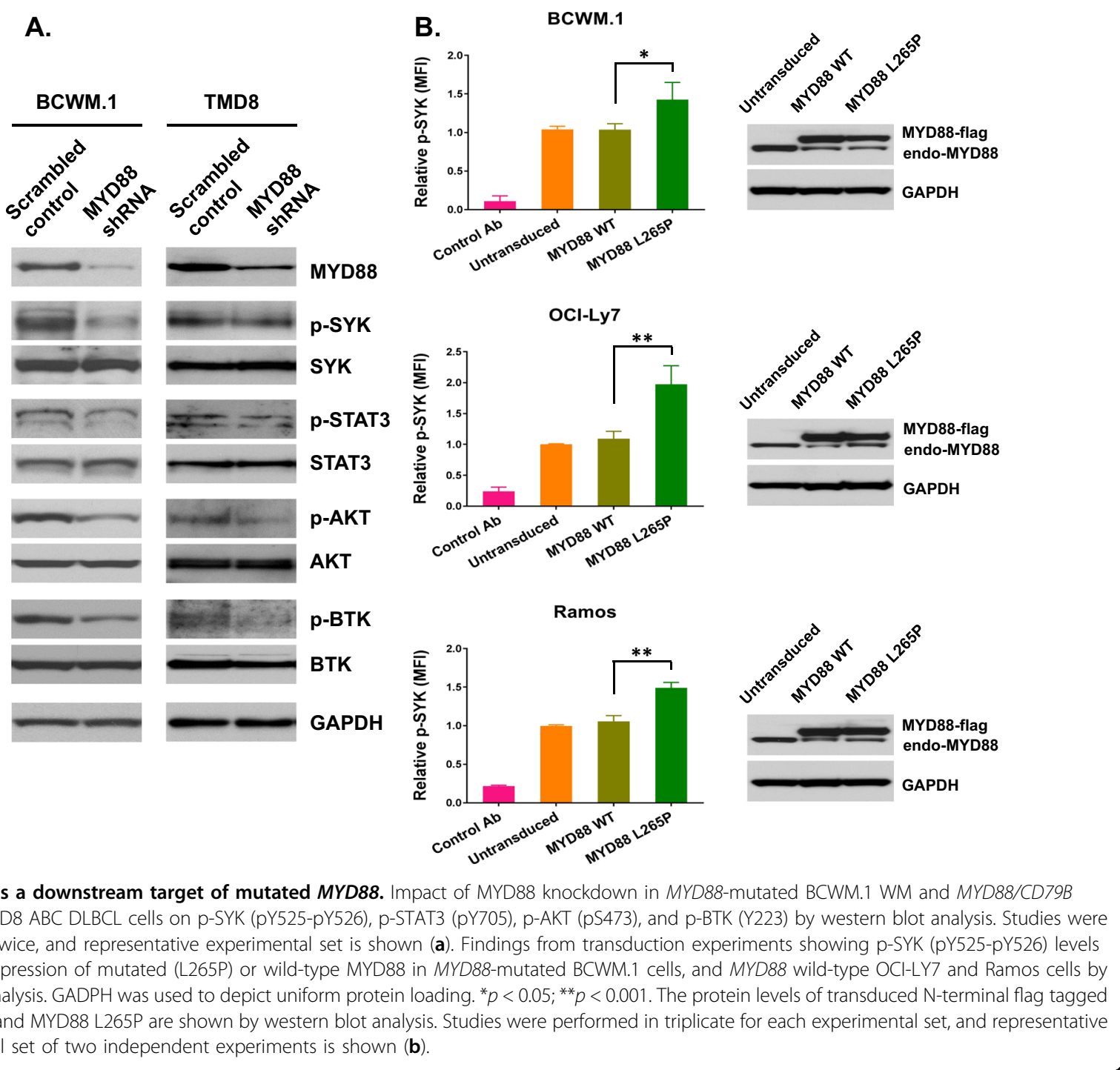

\section{Activated SYK promotes STAT3 and AKT signaling as an intermediary of mutated MYD88}

To explore the downstream events of activated SYK in MYD88-mutated WM cells, we next knocked down SYK using two lentiviral vectors in BCWM.1 cells. Knockdown of SYK reduced the phosphorylation of STAT3 (pY705) and AKT (pS473), while only modest or no changes in BTK, IRAK1 and IRAK4 phosphorylation were observed (Fig. 4a). In addition to these experiments, we treated MYD88-mutated WM and ABC DLBCL cells with tamatinib or entospletinib, and examined changes in the phosphorylation levels of SYK, STAT3, and AKT. As expected, tamatinib as well as entospletinib effectively blocked the phosphorylation of SYK, and its downstream components STAT3 and AKT in MYD88 only mutated WM cells, as well as in ABC DLBCL cell lines carrying both MYD88 and CD79B activating mutations (Fig. 4b).
To further clarify the downstream signaling pathways impacted by SYK in MYD88-mutated WM cells, we engineered BCWM.1 cells to express vector only, wildtype MYD88 or MYD88 L265P and treated them with the SYK inhibitors tamatinib or entospletinib. The increased phosphorylation of SYK (pY525-pY526), as well as the known downstream signaling components STAT3 (pY705) and AKT (pS473) induced by the expression of MYD88 ${ }^{\mathrm{L} 265 \mathrm{P}}$ were blocked by both SYK inhibitors in a dose-dependent manner (Fig. 4c).

\section{SYK supports growth and survival of MYD88-mutated lymphoma cells}

Since SYK activation is directly caused by mutated MYD88 and mediates its downstream STAT3 and AKT signaling, we next sought to clarify the importance of SYK in supporting cell growth and survival in MYD88-mutated 


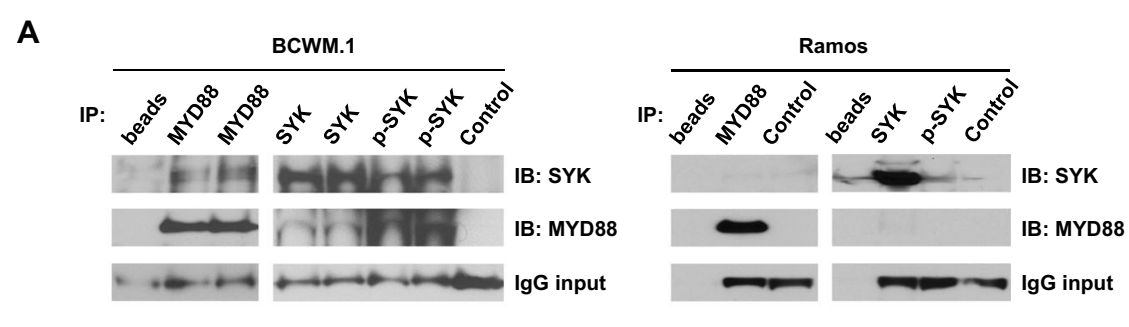

B
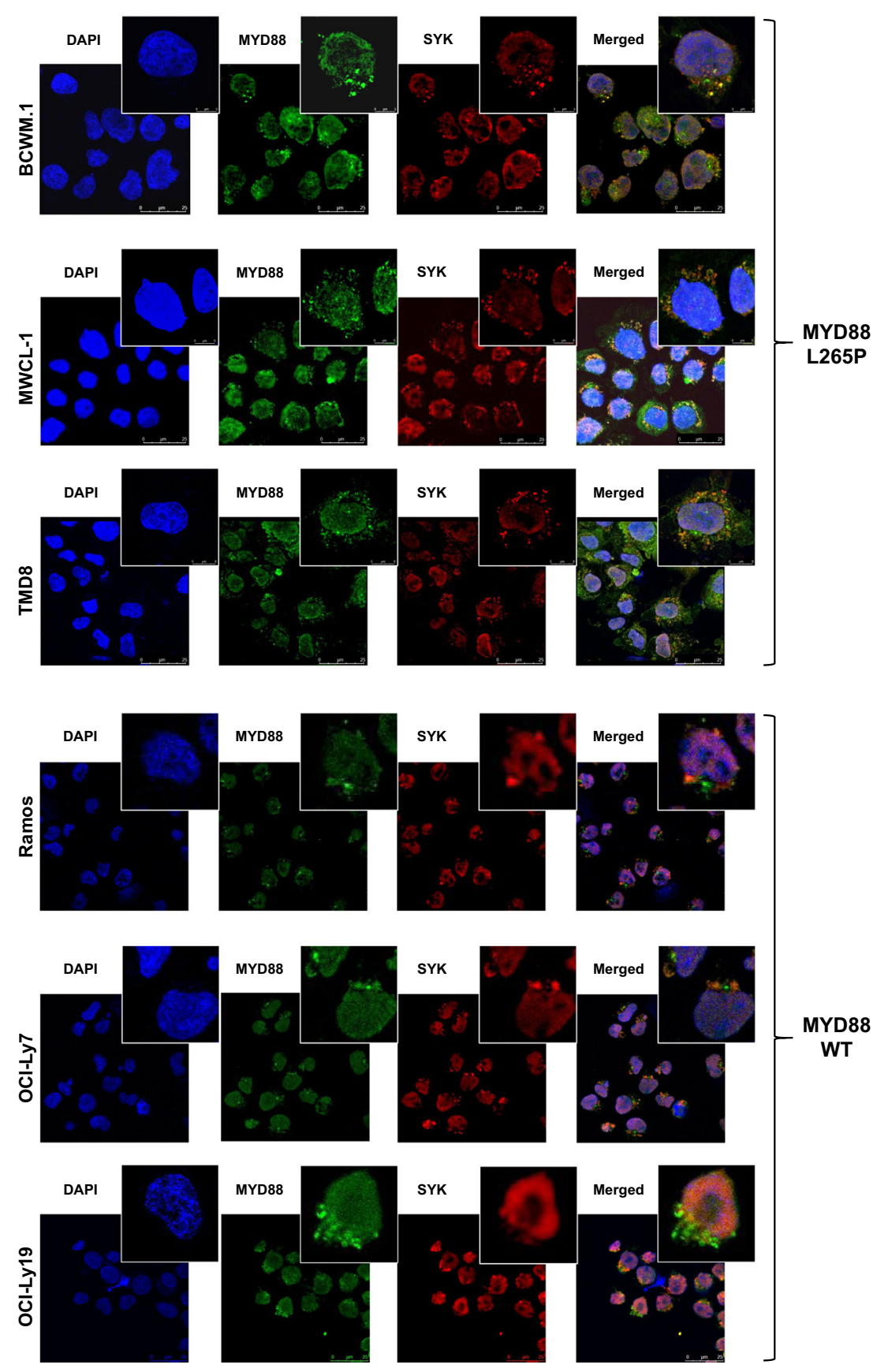

Fig. 3 (See legend on next page.) 
(see figure on previous page)

Fig. 3 Activated SYK is a component of the "Myddosome" signaling complex in MYD88-mutated lymphoma cells. Western blots showing SYK pulldown by MYD88 antibody and MYD88 pulldowns by SYK and p-SYK antibodies in MYD88-mutated and wild-type cells in co-IP experiments. Experiments were run using $2 \mathrm{mg}$ of total protein in duplicated samples, and lgG levels used to demonstrate uniform antibody addition for pulldown experiments (a). Confocal microscopy images showing nuclear staining by DAPI (blue); MYD88 staining by anti-MYD88-Alexa Fluor ${ }^{\circledR} 488$ (green); and SYK staining by anti-SYK-Alexa Fluor ${ }^{\oplus} 647$ (red) antibodies. MYD88 and SYK protein co-localization appears in the cytoplasm (yellow) with a punctate staining pattern following merging of MYD88 and SYK staining images in MYD88-mutated BCWM.1 and MWCL-1 WM cells, and TMD8 ABC DLBCL cells, but not in MYD88 wild-type Ramos Burkitt's lymphoma cells, or OCI-Ly7 and OCI-Ly19 GCB DLBCL cells (b). Studies were performed twice, and representative experimental set is shown.

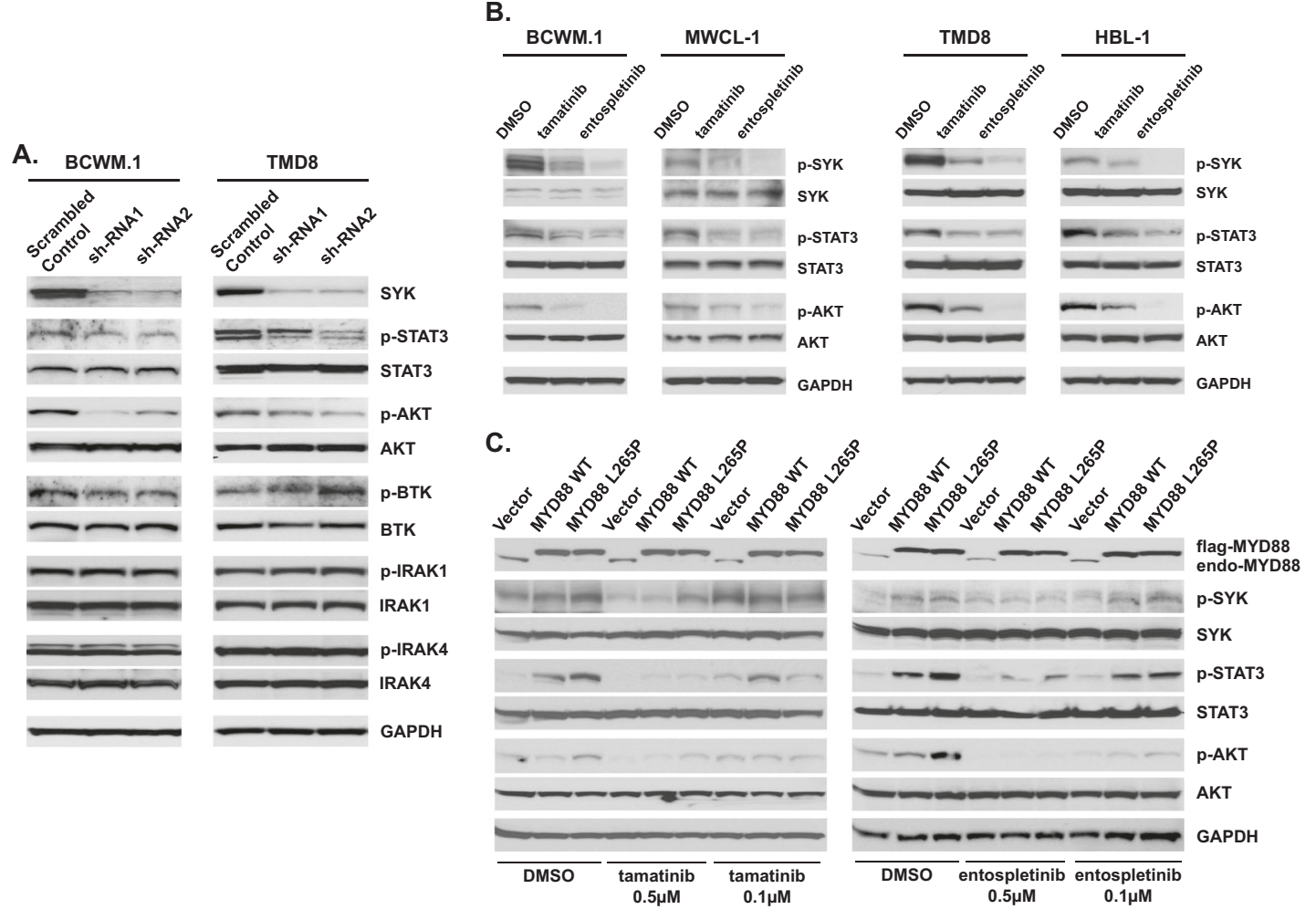

B.

Fig. 4 Activated SYK promotes STAT3 and AKT signaling as an intermediary of mutated MYD88. Impact of SYK knockdown by lentiviral transduction (a) or use of SYK inhibitors tamatinib (TAM) or entospletinib (ENTO) (b) on p-STAT3 (Y705), p-ATK (S473), p-BTK (Y223), p-IRAK1(T209), and p-IRAK4(T345/S346) in MYD88-mutated BCWM.1 WM and MYD88/CD79B mutated TMD8 ABC DLBCL cells. The results from experiments assessing the impact of mutated (L265P) or wild-type MYD88 expression on p-SYK (pY525-pY526), p-STAT3 (pY705), and p-AKT (pS473) in the presence or absence of SYK inhibitors (c). GAPDH was used to depict uniform protein loading. Studies were performed twice, and representative experimental set is shown.

lymphoma cells. We therefore assessed the cell growth and survival of MYD88-mutated WM and ABC DLBCL cells, including MYD88/CD79B mutated TMD8 and HBL-1 cells using CellTiter-Glo ${ }^{\circledR}$ Luminescent cell viability assay following SYK knockdown by lentiviral transduction. GFP-positive cells were sorted on day 5 following lentiviral transduction, and the cell growth and survival were determined every 2 days until day 11 . Compared with scrambled control, knockdown of SYK reduced cell growth and survival in BCWM.1, MWCL-1, and TMD8 cells (Fig. 5a). Moreover, treatment of
MYD88-mutated WM and MYD88/CD79B ABC DLBCL cell lines with tamatinib or entospletinib showed killing at pharmacologically achievable levels for either agent (Fig. 5b) $)^{21,25}$.

\section{The combined inhibition of BTK and SYK produces} synergistic lethality in MYD88-mutated lymphoma cells

Since SYK provides a divergent stream of pro-survival signaling from previously identified mutated MYD88 driven BTK and HCK, both targets of ibrutinib, we next sought to examine the combined effects of ibrutinib and 
A.
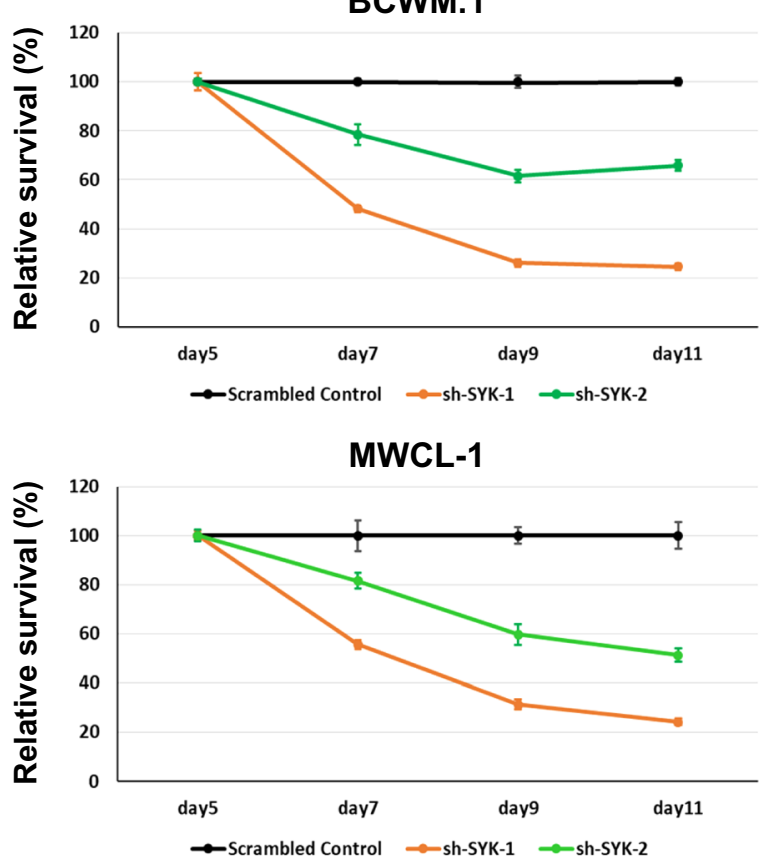

TMD8

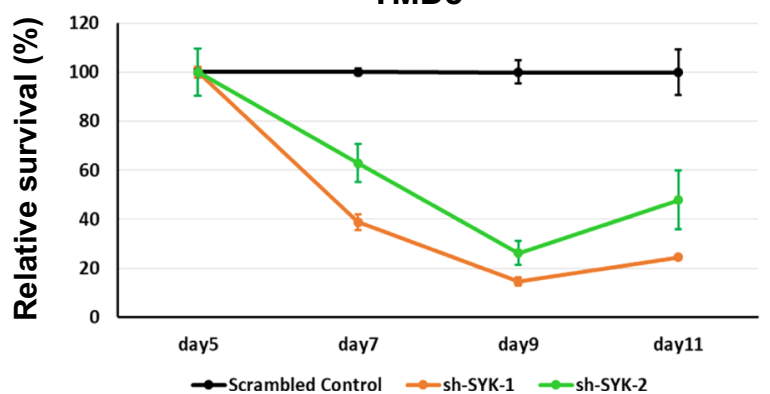

B.

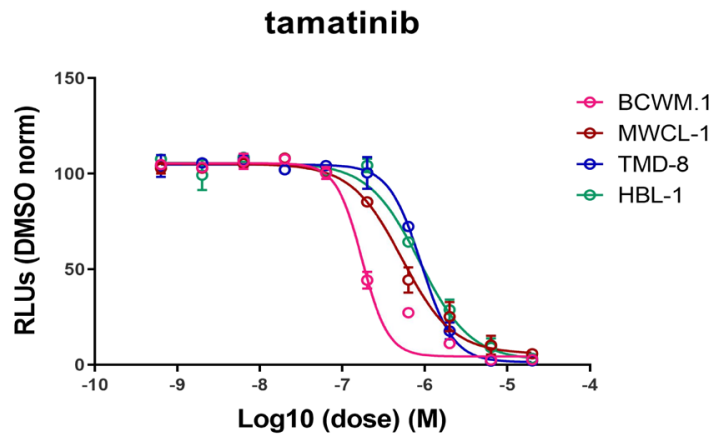

entospletinib

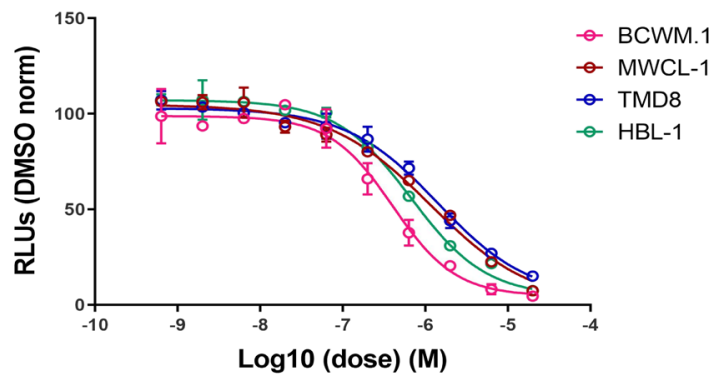

Fig. 5 SYK supports growth and survival of MYD88-mutated lymphoma cells. Impact of SYK knockdown on the cell growth and survival of MYD88-mutated BCWM.1, MWCL-1, and MYD88/CD79B mutated TMD8 ABC DLBCL cells was determined by CellTiter-Glo" luminescent cell viability assay following cell sorting for GFP + cells at day 5 of lentiviral vector transduction. Cell viability was tracked from day 5 to day 11 following lentiviral transduction (a). Dose-response curves for the SYK inhibitors tamatinib and entospletinib in MYD88-mutated WM (BCWM.1, MWCL-1) and MYD88/ CD79B mutated ABC DLBCL (TMD8, HBL-1) cells (b). Studies were performed in triplicate for each experimental set, and representative experimental set of two independent experiments is shown.

either tamatinib or entospletinib. The combination of ibrutinib with either SYK inhibitor produced increased lethality in MYD88-mutated BCWM.1 and MWCL-1 WM cells, as well as MYD88/CD79B mutated TMD8 and HBL-1 ABC DLBCL cells. Combination index $(\mathrm{CI})$ and normalized isobologram analysis indicated ibrutinib and SYK inhibitor interactions were synergistic across nearly all doses that were evaluated (Fig. 6a-d).

\section{Discussion}

Despite the rarity of $B C R$ pathway mutations in WM and their absence in many patients with $A B C$ DLBCL, there are indications that chronic active $\mathrm{BCR}$ signaling is occurring in these entities. In previous studies, we identified that BTK, a downstream contributor to BCR signaling, was activated by mutated MYD88 through HCK and was incorporated into the "Myddosome" signaling complex that supported $\mathrm{NF}_{\mathrm{K}} \mathrm{B}$ pro-survival signaling ${ }^{5,6}$. These findings enabled the investigation and fast-track approval of ibrutinib, a pleiotropic kinase that targets both BTK and HCK for WM. However, the activation of BTK by mutated MYD88 did not provide an explanation for SYK activation, an upstream BCR member that is activated in MYD88-mutated WM patient cells ${ }^{17}$. Moreover, the finding that fostamatinib triggered apoptosis of MYD88-mutated WM cells, suggested an important function for SYK in MYD88 prosurvival signaling ${ }^{20}$. We therefore investigated a role for 


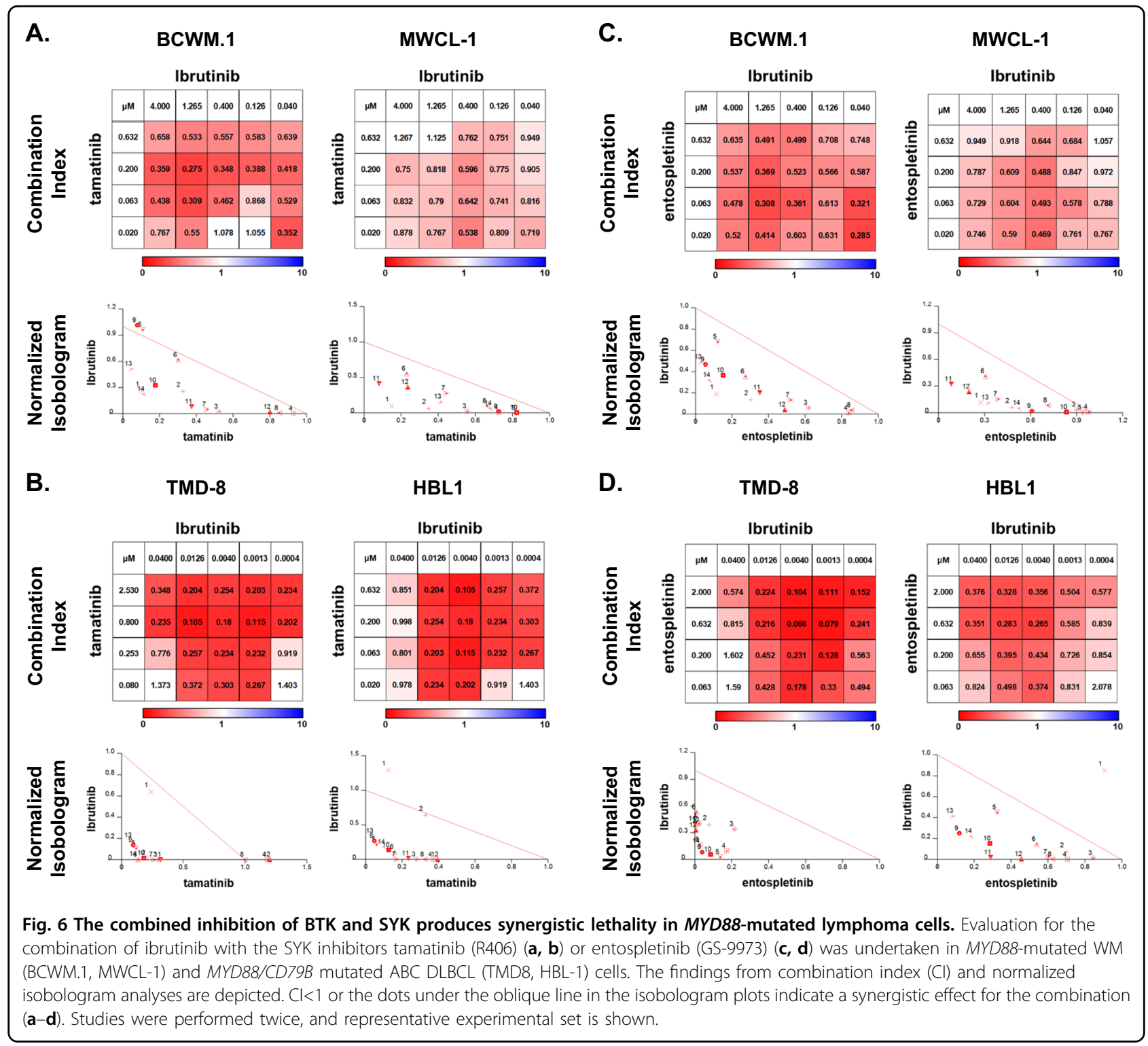

SYK as a mediator of TLR/BCR cross talk in MYD88mutated lymphomas.

We observed that SYK in its active form was incorporated into the "Myddosome" signaling complex by both co-immunoprecipitation and co-localization experiments. Furthermore, by knockdown studies as well as use of SYK inhibitors, we validated the importance of SYK as an essential pro-survival molecule in MYD88-mutated lymphoma cells. The findings are consistent with those recently reported by Phelan et al. ${ }^{26}$, who described a MYD88-TLR9-BCR(IGM) (MY-T-BCR) super complex in MYD88-mutated tumor cells. Importantly, we found that SYK was activated by mutated MYD88, and served as an intermediary to trigger pro-survival STAT3 and AKT, but not BTK signaling. The latter finding is consistent with our earlier work that showed BTK activation was dependent on mutated $M Y D 88^{5}$. Our current observations depict SYK and BTK, classically viewed as hierarchal components of BCR signaling, as "Myddosome" recruits that serve independent roles in propagating MYD88/TLR pathway signaling. Taken together, the findings add to the complexity and reach of mutated MYD88 to drive multiple pro-survival pathways that lead to NFKB, AKT, ERK, and STAT3 activation (Fig. 7).

The identification of SYK as a pro-survival signaling intermediary for mutated MYD88 may also explain the lack of complete responses reported with ibrutinib in MYD88-mutated B-cell malignancies, since SYK unlike BTK or HCK is not a target of these agents ${ }^{6-11}$. Importantly, the combination of SYK inhibitors with ibrutinib yielded synergistic lethality in MYD88-mutated lymphoma cells. These findings provide support for clinical 


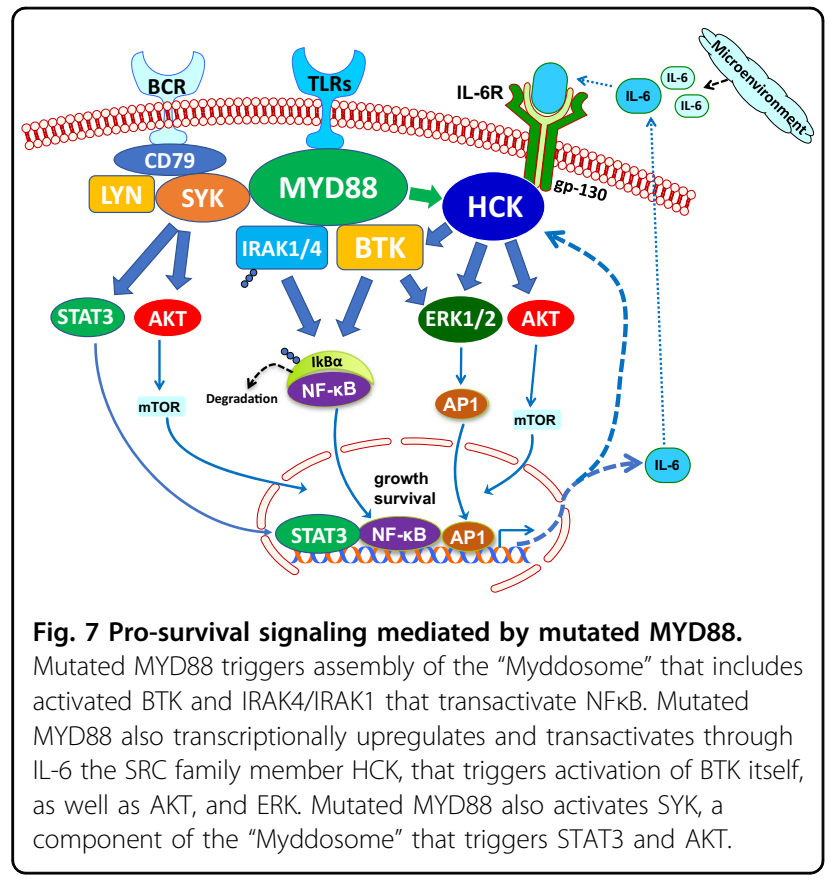

trials combining SYK inhibitors with ibrutinib for MYD88-mutated lymphomas. Such an approach may be particularly suited to patients with both MYD88 and CD79 activating mutations since SYK remained active despite MYD88 inhibition in MYD88/CD79 doublemutated ABC cell lines. SYK inhibitors would therefore block upstream SYK activation mediated by activating CD79 mutations in these double-mutated tumors. Several SYK inhibitors, including fostamatinib, entospletinib, TAK-659, and HMPL-23 are currently in development for hematological and oncological indications. Fostamatinib was recently approved for the treatment of chronic ITP and showed significant single agent activity in previously treated CLL ${ }^{27}$. Entospletinib, a more selective SYK inhibitor, has also shown notable activity in previously treated CLL, with good tolerance and is currently being evaluated in combination with obinutuzumab in CLL, SLL, and NHL (NCT 03010358) ${ }^{22,28}$. The SYK inhibitor TAK-659 has also shown activity and good tolerance in FL, and DLBCL, including transformed cases ${ }^{29}$. A study evaluating TAK-695 in combination with chemotherapy is currently underway in high-risk DLBCL (NCT 03742258). Responses to the SYK inhibitor HMPL-3 have also been observed across multiple B-cell lymphomas, including $\mathrm{WM}^{30}$.

In summary, we have identified activated SYK as an integral component of the mutated MYD88 signaling apparatus, and an intermediary for AKT and STAT3 prosurvival signaling. Combined use of ibrutinib and SYK inhibitors produces synergistic lethality in MYD88mutated B cells, including those with accompanying CD79 activating mutations. The findings provide a framework for the clinical investigation of ibrutinib with SYK inhibitors in MYD88-mutated lymphomas.

\section{Acknowledgements}

The authors gratefully acknowledge the generous support of Peter Bing M.D., the David and Janet Bingham Research Fund and Yang Family Fund of the International Waldenstrom's Macroglobulinemia Foundation, the Leukemia and Lymphoma Society (Grant: R6507-18), the NIH SPORE in Multiple Myeloma (Grant: 2P50CA100707-16A1), the Edward and Linda Nelson Fund for WM Research, the Kerry Robertson Fund for WM Research, the Bauman Family Trust, and the WM patients who provided samples for these studies.

\section{Author details}

'Bing Center for Waldenstrom's Macroglobulinemia, Dana Farber Cancer Institute and Harvard Medical School, Boston, MA, USA. ${ }^{2}$ Department of Medical Oncology, Dana Farber Cancer Institute and Harvard Medical School, Boston, MA, USA. ${ }^{3}$ Lymphoma Service, Memorial Sloan Kettering Cancer Center, New York, NY, USA. ${ }^{4}$ Department of Biological Chemistry and Molecular Pharmacology, Harvard Medical School, Boston, MA, USA

\section{Author contributions}

G.Y., M.M., and S.P.T. conceived and designed the experiments and wrote the paper. M.L.P. and K.A. provided data in support of BCR signaling in WM. J.W., S.J B., and N.S.G. provided input on signaling studies. G.Y. and M.M. performed the data analysis; M.M. performed PhosFLow, immunoblotting and coimmunoprecipitation assays; J.G.C. and M.M. performed drug treatment and cell viability assessments. X.L. performed lentiviral experiments. Z.R.H., G.C., and C.J. provided informatic support. L.X., N.T., M.G.D., M.L.G., and A.K. prepared samples. J.J.C., C.J.P., K.M., T.D., K.M., A.K., J.G., and S.P.T. provided patient care, obtained consent and samples.

\section{Conflict of interest}

S.T., Z.R.H., M.L.P., J.J.C., and G.Y. have received research funding, consulting fees, and/or honoraria from Pharmacyclics Inc., and Janssen Oncology Inc.

\section{Publisher's note}

Springer Nature remains neutral with regard to jurisdictional claims in published maps and institutional affiliations.

Received: 26 August 2019 Revised: 30 December 2019 Accepted: 13 January 2020

Published online: 31 January 2020

\section{References}

1. Treon, S. P. et al. MYD88 L265P somatic mutation in Waldenstrom's macroglobulinemia. N. Engl. J. Med. 367, 826-833 (2012).

2. Ngo, V. N. et al. Oncogenically active MYD88 mutations in human lymphoma. Nature 470, 115-119 (2011).

3. Loiarro, M. et al. Mutational analysis identifies residues crucial for homodimerization of myeloid differentiation factor 88 (MyD88) and for its function in immune cells. J. Biol. Chem. 288, 30210-30222 (2013).

4. Avbelj, M. et al. Activation of lymphoma-associated MyD88 mutations via allostery-induced TIR-domain oligomerization. Blood 124, 3896-3904 (2014).

5. Yang, G. et al. A mutation in MYD88 (L265P) supports the survival of lymphoplasmacytic cells by activation of Bruton tyrosine kinase in Waldenstrom macroglobulinemia. Blood 122, 1222-1232 (2013).

6. Yang, G. et al. HCK is a survival determinant transactivated by mutated MYD88, and a direct target of ibrutinib. Blood 127, 3237-3252 (2016).

7. Treon, S. P. et al. Ibrutinib in previously treated Waldenstrom's macroglobulinemia. N. Engl. J. Med. 372, 1430-1440 (2015).

8. Wilson, W. H. et al. Targeting B cell receptor signaling with ibrutinib in diffuse large B cell lymphoma. Nat. Med. 21, 922-926 (2015).

9. Grommes, C. et al. Ibrutinib unmasks critical role of bruton tyrosine kinase in primary CNS lymphoma. Cancer Discov. 7, 1018-1029 (2017).

10. Lionakis, M. S. et al. Inhibition of B cell receptor signaling by ibrutinib in primary CNS lymphoma. Cancer Cell 31, 833-843 (2017). 
11. Yamada, S., Ishida, Y., Matsuno, A. \& Yamazaki, K. Primary diffuse large B-cell lymphomas of central nervous system exhibit remarkably high prevalence of oncogenic MYD88 and CD79B mutations. Leuk Lymphoma 56, 2141-2145 (2015).

12. Wang, J. Q. et al. Synergistic cooperation and crosstalk between MYD88 (L265P) and mutations that dysregulate CD79B and surface lgM. J Exp Med. 214, 2759-2776 (2017).

13. Hunter, Z. R. et al. The genomic landscape of Waldenstrom macroglobulinemia is characterized by highly recurring MYD88 and WHIM-like CXCR4 mutations, and small somatic deletions associated with B-cell lymphomagenesis. Blood 123, 1637-1646 (2014).

14. Poulain, S. et al. Genomic landscape of CXCR4 mutations in Waldenström macroglobulinemia. Clin. Cancer Res. 22, 1480-1488 (2016).

15. Varettoni, M. et al. Pattern of somatic mutations in patients with Waldenström macroglobulinemia or lgM monoclonal gammopathy of undetermined significance. Haematologica 102, 2077-2085 (2017).

16. Jiménez, C. et al. From Waldenström's macroglobulinemia to aggressive diffuse large B-cell lymphoma: a whole-exome analysis of abnormalities leading to transformation. Blood Cancer J. 7, e591 (2017).

17. Argyropoulos, K. V. et al. Clonal B cells in Waldenstrom's macroglobulinemia exhibit functional features of chronic active B-cell receptor signaling. Leukemia 30, 1116-1125 (2016).

18. Paiva, B. et al. The cellular origin and malignant transformation of Waldenstrom macroglobulinemia. Blood 125, 2370-2380 (2015).

19. Davies, R. E. et al. Chronic active B-cell-receptor signalling in diffuse large B-cell lymphoma. Nature 463, 88-92 (2010).

20. Kuiatse, I. et al. Targeting the spleen tyrosine kinase with fostamatinib as a strategy against Waldenström macroglobulinemia. Clin. Cancer Res. 21 2538-2545 (2015).
21. Baluom, M., Grossbard, E. B., Mant, T. \& Lau, D. T. W. Pharmacokinetics of fostamatinib, a spleen tyrosine kinase (SYK) inhibitor, in healthy human subjects following single and multiple oral dosing in three phase I studies. Br. J. Clin. Pharmacol. 76, 78-88 (2013).

22. Sharman, J. et al. An open-label phase 2 trial of entospletinib (GS-9973), a selective spleen tyrosine kinase inhibitor, in chronic lymphocytic leukemia. Blood 125, 2336-2343 (2015).

23. $\mathrm{Xu}$, L. et al. MYD88 L265P in Waldenstrom macroglobulinemia, immunoglobulin $\mathrm{M}$ monoclonal gammopathy, and other B-cell lymphoproliferative disorders using conventional and quantitative allele-specific polymerase chain reaction. Blood 121, 2051-2058 (2013).

24. Chou, T. C. Drug combination studies and their synergy quantification using the Chou-Talalay method. Cancer Res. 70, 440-446 (2010).

25. Ramanathan, S., DiPaolo, J. A., Doan, T. \& Burge, D. Single and multiple doseranging evaluation of safety, pharmacokinetics, and pharmacodynamics of GS9973, a novel pSYK inhibitor. Cancer Res. 73, 32 (2013).

26. Phelan, J. D. et al. A multiprotein supercomplex controlling oncogenic signaling in lymphoma. Nature 560, 387-391 (2018).

27. Friedberg, J. W. et al. Inhibition of SYK with fostamatinib disodium has significant clinical activity in non-Hodgkin lymphoma and chronic lymphocytic leukemia. Blood 115, 2578-2585 (2010).

28. Sharman, J. P. et al. Phase 2 trial of entospletinib (GS-9973), a selective Syk inhibitor, in indolent non-Hodgkin's lymphoma (iNHL). Blood 126, 1545 (2015).

29. Kaplan, J. B. et al. Updated results from a Phase 1 study of TAK-659, an investigational and reversible SYK inhibitor, in patients with advanced solid tumor or lymphoma malignancies. Blood 128, 624 (2016).

30. Zhu, J. et al. Preliminary results from a phase 1 study of HMPL-523, a highly selective Syk inhibitor, in Chinese patients with mature B-cell lymphomas. Blood 132, 5324 (2018). 